\title{
The clinical outcomes and biomarker features of severe sepsis/ septic shock with severe neutropenia: a retrospective cohort study
}

\author{
Fan Yin ${ }^{1 \#}$, Yue-Ling $\mathrm{Xi}^{1 \#}$, Ying Wang ${ }^{1}, \mathrm{Bi}-\mathrm{Ru} \mathrm{Li}^{1}$, Juan Qian ${ }^{1}$, Hong Ren ${ }^{1}$, Jian Zhang ${ }^{1}$, Hong-Zhi Tang ${ }^{2}$, \\ Bo-Tao Ning'
}

${ }^{1}$ Department of Pediatric Intensive Care Unit, Shanghai Children's Medical Center, School of Medicine, Shanghai Jiao Tong University, Shanghai, China; ${ }^{2}$ Department of State Key Laboratory of Microbial Metabolism, and School of Life Sciences \& Biotechnology, Shanghai Jiao Tong University, Shanghai, China

Contributions: (I) Conception and design: BT Ning; (II) Administrative support: BT Ning; (III) Provision of study materials or patients: BT Ning; (IV) Collection and assembly of data: F Ying, YL Xi; (V) Data analysis and interpretation: F Ying, YL Xi; (VI) Manuscript writing: All authors; (VII) Final approval of manuscript: All authors.

\#These authors contributed equally to this work.

Correspondence to: Bo-Tao Ning. Shanghai Children's Medical Center of Shanghai Jiao Tong University, 1678, Dongfang Road, Shanghai, China. Email: ningbotao@126.com.

Background: Severe sepsis/septic shock with severe neutropenia often leads to poor prognosis. However, it is unknown if severe neutropenia is associated with different clinical outcomes and biomarker features in severe sepsis/septic patients.

Methods: This retrospective cohort study enrolled 141 severe sepsis/septic shock patients admitted to intensive care unit of Shanghai Children's Medical Center between January 2015 and November 2019. Patients were followed up for the development of ventilation support, the use of vasoactive drugs, continuous renal replacement therapy (CRRT) procedure, and mortality. Biomarkers that reflect the level of inflammation in severe sepsis/septic shock patients with neutropenia were compared to that in patients without neutropenia.

Results: Of 141 patients enrolled, 54 patients suffered from severe sepsis/septic shock with severe neutropenia. In patients with severe sepsis/septic shock, severe neutropenia as a complication was an independent risk factor for the use of vasoactive drugs (RR 9.796; 95\% CI: 3.774, 25.429; P<0.001), but not for ventilation support (RR 0.157; 95\% CI: 0.06, 0.414; $\mathrm{P}<0.001)$, CRRT procedure (RR 1.032; 95\% CI: $0.359,2.969 ; \mathrm{P}=0.953$ ) or 28-day mortality (RR 1.405; 95\% CI: $0.533,3.708 ; \mathrm{P}=0.492$ ). Severe sepsis/septic patients with severe neutropenia had a higher plasma level of the following biomarkers: c-reaction protein (CRP) (180.5 vs. $121 \mathrm{mg} / \mathrm{mL}, \mathrm{P}<0.001)$, procalcitonin (PCT) (12.15 vs. $2.7 \mathrm{ng} / \mathrm{mL} ; \mathrm{P}=0.005)$, interleukin (IL)-6 (316.83 vs. 55.77 pg/mL, $\mathrm{P}<0.001$ ), IL-10 (39.165 vs. 10.09 pg/mL, $\mathrm{P}<0.001$ ), interferon (IFN)- $\gamma(6.155$ vs. $3.71 \mathrm{pg} / \mathrm{mL}, \mathrm{P}=0.016)$, and the percentage of regulatory T cells (Tregs) $(2.7 \%$ vs. $2.09 \%, \mathrm{P}=0.003)$. Based on the receiver operating characteristic curves, IL-10 exhibited high specificity $(79.4 \%)$ in evaluating the prognosis of septic patients with neutropenia.

Conclusions: In patients with severe sepsis/septic shock, being complicated with severe neutropenia is associated with higher proportion of using vasoactive drugs, and those patients tend to have higher plasma levels of IL-6, IL-10, IFN- $\gamma$ and percentage of Treg.

Keywords: Severe sepsis; septic shock; neutropenia; risk ratio; cytokines.

Submitted Aug 06, 2020. Accepted for publication Jan 04, 2021.

doi: $10.21037 / \mathrm{tp}-20-230$

View this article at: http://dx.doi.org/10.21037/tp-20-230 


\section{Introduction}

Sepsis refers to a life-threatening organ dysfunction caused by a host's dysregulation of infection. Currently, apart from life support therapy, the definitive therapy of sepsis is so limited that it is the leading cause of death in intensive care unit (ICU) $(1,2)$. Immune disorder caused by malignant tumor, secondary immunodeficiency, or the use of immunosuppressants is increasingly common in ICU. In PICU, myelosuppression and neutropenia caused by chemotherapy of leukemia or other tumors increase the risk of infection and death in patients (3-5). Neutropenia has been proven to be an independent risk factor for sepsis $(6,7)$. Patients with severe neutropenia have lower levels of neutrophils in the periphery. Since neutrophils mediate a variety of pathophysiological processes in sepsis, it is essential to understand whether severe sepsis/septic shock patients with severe neutropenia show distinct clinical and biomarker features.

Acute kidney injury (AKI) in ICU caused by sepsis is often treated by continuous renal replacement therapy (CRRT) in advanced stages (8). When severe sepsis patients get worse and have oxygenation disorders, airway protection and mechanical ventilation support are important treatment measures (9). The use of vasoactive drugs such as norepinephrine can help correct circulatory disorders and improve tissue perfusion in patients with sepsis (10). CRRT, ventilation and vasoactive drugs are important organ support methods, which reflect the dysfunction of main organs and indicate the severity of sepsis. The high level of CRP and PCT, as classic acute phase proteins during sepsis, is valuable for sepsis diagnosis $(11,12)$. In this study, the cytokines were determined after our discussion, including interleukin (IL)-2, IL-6, tumor necrosis factor (TNF)- $\alpha$, which are major pro- and anti-inflammatory cytokines and have been proven to reflect the severity and prognosis of sepsis (13-16). Regulatory T cells (Tregs) plays a role in maintaining the immunologic homeostasis and tolerance, so the percentage of Tregs reflects the immune status during sepsis $(17,18)$. However, whether neutropenia is associated with distinct clinical and biomarker features in patients with severe sepsis remains unclear.

We aim to evaluate clinical and biomarker features in severe sepsis/septic shock children with severe neutropenia. This retrospective cohort study may provide a new perspective for clinical options in sepsis treatment.

We present the following article in accordance with the STROBE reporting checklist (available at http://dx.doi. org/10.21037/tp-20-230).

\section{Methods}

\section{Study subjects}

This retrospective cohort study enrolled 141 cases of patients (87 males and 54 females) from January 2015 through November 2019, and were hospitalized at Department of Pediatric Intensive Care Unit, Shanghai Children's Medical Center, School of Medicine Affiliated to Shanghai Jiao Tong University. Inclusion criteria were as follow: aged from 1 month to 18 years old; diagnosed with severe sepsis or septic shock according to American College of Chest Physicians/ Society of Critical Care Medicine consensus criteria (19); complicated with sepsis-induced organ dysfunction, tissue hypoperfusion or sepsis-induced hypotension. Patients who were diagnosed with hemophagocytic syndrome or received Chimeric Antigen Receptor T-Cell Immunotherapy (CAR-T) were excluded. The study was performed in accordance with the Declaration of Helsinki (2013 revision) and was approved by the Research Ethics Committee of the Shanghai Children's Medical Center of Shanghai Jiao Tong University (Ethical code: SCMCIRBW2020049). Individual consent for this retrospective analysis was waived.

\section{Data collection}

Research personnel used standardized case report forms to collect clinical and laboratory data of enrolled subjects from the electronic medical record (EMR) system. General information (including age, gender, underlying disease), PRISM III score and laboratory data (blood culture results, serum inflammatory protein, cytokines, and percentage of Tregs) were recorded at PICU admission.

\section{Exposure and outcome definitions}

Severe neutropenia was defined as an absolute neutrophil count in peripheral blood less than 100/microliter on PICU admission (20). Research personnel had followed up the use of mechanical ventilation, vasoactive drugs and CRRT of the patients for 7 days from admission. Organ support treatment was given to the patients when they developed AKI, acute respiratory distress syndrome (ARDS), or severe circulatory disturbance. All medical decisions were made by experienced physicians. Mortality was defined at 28 days after admission.

\section{Sample collection and laboratory testing}

Peripheral blood samples of all children were collected on 
the day of PICU admission. CRP and PCT were measured by enzyme-linked immunosorbent assays (ELISA). Cytokines, including IL-2, IL-4, IL-6, IL-10, TNF- $\alpha$, IFN- $\gamma$, IL-17A were determined with cytometric bead array (CBA). Detection reagent was $\mathrm{BD}^{\mathrm{TM}} \mathrm{CBA}$ human $\mathrm{T}$ helper cell 1/2 (Th1/Th2) cytokine kit II (BD Biosciences, San Jose, CA). Tregs were identified based on the expression of CD4, CD25 and CD127, and results were expressed as the proportion of the total number of lymphocytes.

\section{Statistical analysis}

Continuous data were expressed as median and range. Between groups, the differences were tested for significance using the Student's $t$-test or Manne-Whitney $U$ test. The enumeration data were expressed by rate. The Pearson's chi-square test and Fisher's exact test were used. It was considered to be statistically significant when a two-sided $\mathrm{P}$ value was smaller than 0.05 . We also checked the correlation between severe neutropenia and the use of mechanical ventilation, vasoactive drugs, CRRT within 7 days after PICU admission and 28-day mortality using multivariable logistic regression. Possible confounders related to exposure or outcome were included in adjusted models, including age, male and severity of illness measured by Pediatric risk of mortality III (PRISM III) score. According to clinical experience and literature, incidence of the use of mechanical ventilation vasoactive drugs and CRRT is $37 \%$. One hundred forty-one subjects were enrolled in the final analysis, we estimated $80 \%$ power to detect $10 \%$ difference in outcomes. Receiver operating characteristic curves (ROC curves) were applied to determine the sensitivities and specificities for biomarkers measurements in sepsis patients. The area under the curve and the optimal cut-off value were also calculated. The value of the cut-off point corresponding to the largest Youden's index is used as cut off value. All statistical analyses were performed with SPSS software, version 20.0 (SPSS Inc., Chicago, IL, USA).

\section{Results}

\section{Clinical characteristics}

A total of 141 patients with severe sepsis or septic shock were collected and were divided into severe neutropenic group $(n=54)$ and non-neutropenic group $(n=87)$. The mean age (range) of patients was $8(0.2-17)$ years old in the severe neutropenic group and $7.9(0.1-17)$ years old in non-neutropenic group. Among the underlying diseases of these severe neutropenic subjects, the most commonly was leukemia $(n=34,63 \%)$, followed by lymphoma $(n=5$, $9 \%)$, aplastic anemia $(n=4,7.5 \%)$, solid tumor $(n=4,7.5 \%)$, biliary atresia $(n=1,2 \%)$ and congenital heart disease $(n=1,2 \%)$. About $9 \%(n=5)$ of subjects had no underlying diseases. There were 11 subjects tested positive for blood culture $\left(\mathrm{G}^{+}: 1\right.$ vs. $\mathrm{G}^{-}: 9 v$ s. fungi:1) in the severe neutropenic group, while there were $21\left(\mathrm{G}^{+}: 12 v s . \mathrm{G}^{-}: 8\right.$ vs. fungi:1) in non-neutropenic group. There were significant differences between the two groups in blood culture results $(\mathrm{P}=0.016)$ and PRISM III $(\mathrm{P}<0.001)$ scores, but not in the length of ICU stay (Table 1).

Compared with non-neutropenic subjects, severe neutropenic subjects were more likely to receive vasoactive drugs treatment $(74.1 \%$ vs. $29.9 \%, \mathrm{P}<0.001)$ while less likely to receive mechanical ventilation $(46.3 \%$ vs. $72.4 \%$, $\mathrm{P}=0.002)$. 28-day morality and the use of CRRT showed no obvious differences between the two groups (Table 1). Both in adjusted or unadjusted regression models, neutropenia was a risk factor for patients with severe sepsis or septic shock to receive vasoactive drugs [adjusted: 9.796 (3.774, 25.429)]. On the contrary, neutropenia was a protective factor for receiving ventilation [adjusted: 0.157 (0.06, 0.414)]. The risk of 28-day mortality and receiving CRRT within 7 days from PICU admission were not significantly different between the two groups in adjusted or unadjusted regression models (Tables 1,2).

\section{Biomarker features analysis}

Serum levels of CRP, PCT, IL-6, IL-10 and IFN- $\gamma$ and the percentage of Tregs, increased significantly in severe neutropenic group (CRP 180.5 vs. $121 \mathrm{mg} / \mathrm{mL}, \mathrm{P}<0.001$; PCT 12.15 vs. $2.7 \mathrm{ng} / \mathrm{mL}, \mathrm{P}=0.005$; IL-6 316.83 vs. $55.77 \mathrm{pg} / \mathrm{mL}, \mathrm{P}<0.001$; IL-10 39.165 vs. $10.09 \mathrm{pg} / \mathrm{mL}$, $\mathrm{P}<0.001$; IFN- $\gamma 6.155$ vs. $3.71 \mathrm{pg} / \mathrm{mL}, \mathrm{P}=0.016$; Tregs $2.7 \%$ vs.2. 09\%, $\mathrm{P}=0.003$ ), while serum IL-17A showed a different trend (13.855 vs. 21.2, $\mathrm{P}=0.032)$. Serum IL-2, IL-4 and TNF- $\alpha$ levels did not vary by neutropenic status (Table 3). Subgroup analysis based on patient outcome showed that serum IL-10 level of the dead was significantly higher than that of survivors in severe neutropenic group. Further subgroup analysis failed to find any other biomarkers changing along with patients' survival status (Table 3).

To analyze whether severe neutropenia influences the biomarkers features of patients with distinct outcomes, all patients enrolled in the cohort were divided into death 
Table 1 The clinical characteristics and of the study subjects

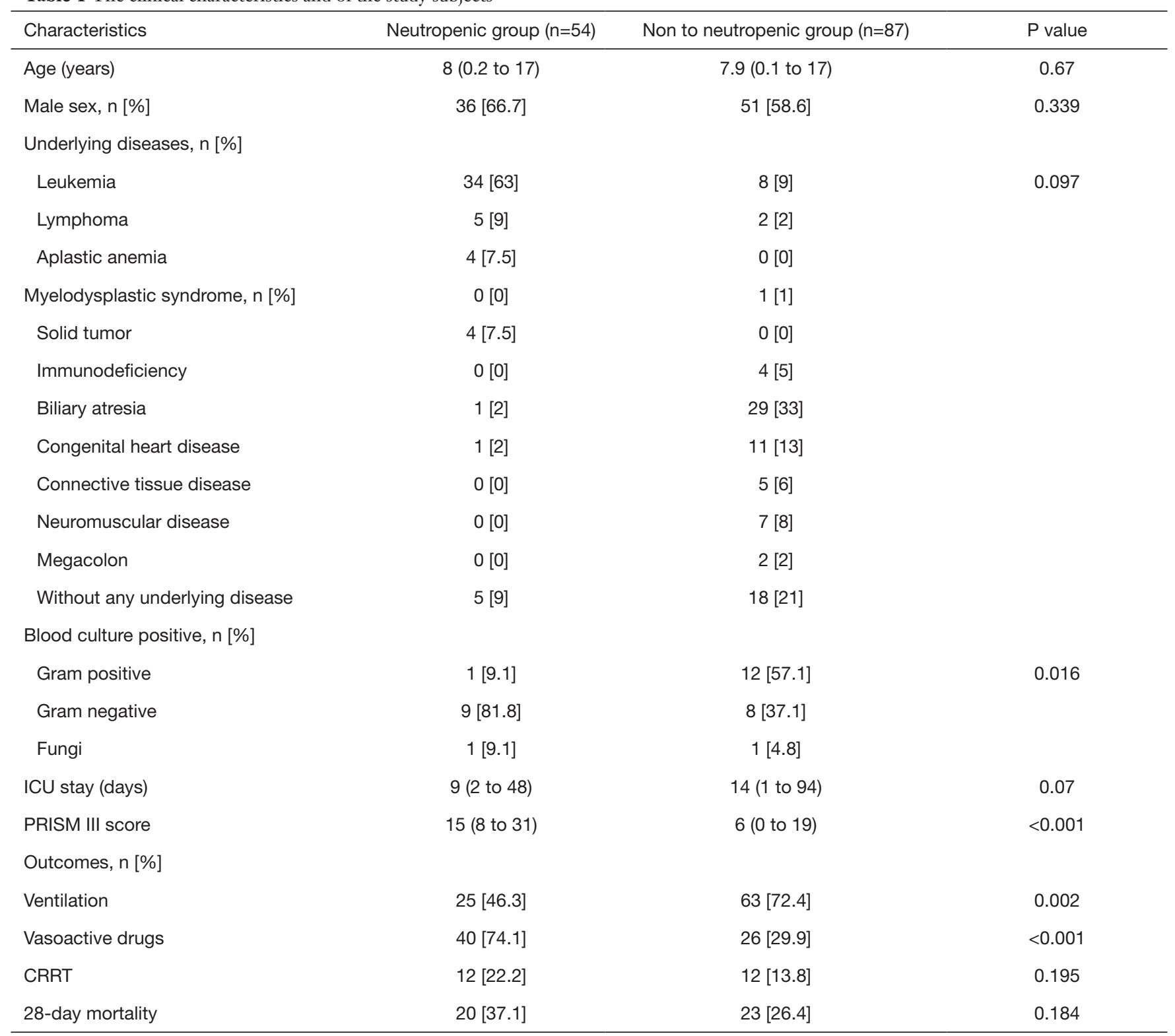

ICU, intensive care unit; CRRT, continue renal replacement therapy; PRISM, pediatric risk of mortality.

Table 2 Associations of neutropenic severe sepsis/septic shock with the use of ventilation, the use of vasoactive drugs, CRRT procedure and 28day mortality

\begin{tabular}{|c|c|c|c|c|c|c|}
\hline Clinical outcomes & \multicolumn{3}{|c|}{ Unadjusted } & \multicolumn{3}{|c|}{ Adjusted } \\
\hline Ventilation & 0.328 & $0.161-0.669$ & 0.002 & 0.157 & $0.06-0.414$ & $<0.001$ \\
\hline Vasoactive drugs & 6.703 & $3.128-14.367$ & $<0.001$ & 9.796 & $3.774-25.429$ & $<0.001$ \\
\hline CRRT & 1.786 & $0.737-4.326$ & 0.199 & 1.032 & $0.359-2.969$ & 0.953 \\
\hline
\end{tabular}

$\mathrm{Cl}$, confidence interval; RR, relative risk; CRRT, continuous renal replacement therapy. 


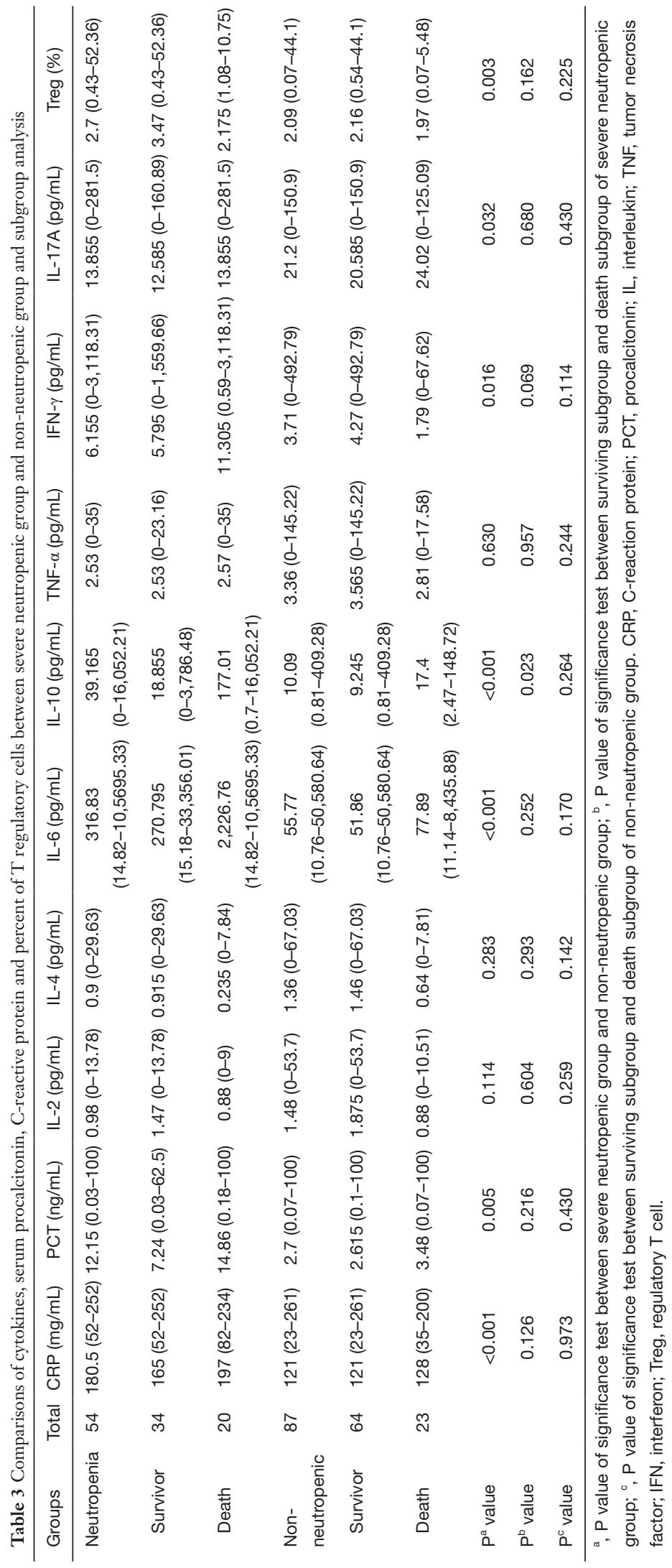


group ( $\mathrm{n}=43$ ) and survival group ( $\mathrm{n}=98)$. Serum IL-6 and IL10 levels were significantly higher in death group compared with survival group (IL-6: 229.73 vs. $74.675 \mathrm{pg} / \mathrm{mL}$, $\mathrm{P}=0.032$; IL-10: 21.92 vs. $13.125 \mathrm{pg} / \mathrm{mL}, \mathrm{P}=0.012$ ). Other biomarkers showed no obvious difference between two groups (Table 4). Further subgroup analysis showed that compared with the non-neutropenic subgroup, the severe neutropenic subgroup had a significant higher level of CRP, PCT, IL-6, IL-10 and \% Treg in survival group (165 vs. $121, \mathrm{P}=0.028 ; 7.24$ vs. $2.615, \mathrm{P}=0.032 ; 270.795$ vs. 51.86, $\mathrm{P}<0.001,18.855$ vs. $9.245, \mathrm{P}=0.009 ; 3.47 \%$ vs. $2.16 \%$, $\mathrm{P}=0.004)$. The level of CRP, IL-6, IL-10 and IFN- $\gamma$ were significantly higher in neutropenic subgroup of death group (197 vs. $128, \mathrm{P}=0.002 ; 2226.76$ vs. 77.89, $\mathrm{P}=0.01 ; 177.01$ vs. 17.4, $\mathrm{P}=0.002 ; 11.305$ vs. $1.79, \mathrm{P}=0.011$ ) (Table 4).

\section{Receiver operating characteristic curve analysis}

To further evaluate the ability of selected biomarkers in assessing the prognosis of patients with different characteristics, we established a ROC curve. For patients with severe sepsis or septic shock, the areas under the curve (AUC) for IL-6 and IL-10 were 0.613 and 0.633 respectively. When setting cut-off points for IL- 6 and IL-10 at 143.255 and $120.26 \mathrm{pg} / \mathrm{mL}$, the sensitivity was $60.5 \%$ and $37.2 \%$, with specificity $62.2 \%$ and $90.8 \%$, respectively for predicting mortality. For severe sepsis/septic shock patients with severe neutropenia, the AUC for IL-10 were 0.687 and the Cut-off value were 110.685 . Accordingly, the sensitivity and specificity were $65 \%$ and $79.4 \%$ respectively (Table 5).

\section{Discussion}

It was more difficult to treat the patients with severe sepsis/ septic shock when they were complicated with neutropenia. In this study, we analyzed the characteristics of clinical outcomes and biomarkers in this population. For patients with severe sepsis/septic shock, being complicated with severe neutropenia is a risk factor of vasoactive drugs using, but a protective factor of mechanical ventilation receiving. Severe neutropenia is not concerned with death or receiving CRRT. Apart from IL-2, IL-4 and TNF-a, other biomarkers increased significantly in severe sepsis/ septic shock patients with severe neutropenia. In particular, IL-6 and IL-10 also showed significant differences between subgroups. ROC curve showed that IL-10 had a high specificity in predicting mortality of severe sepsis/septic shock patients with severe neutropenia.
Severe neutropenia increases the need of vasoactive drugs in severe sepsis/septic shock patient, as severe neutropenia impairs elimination of pathogens and function of defensive barrier, leading to secondary infection and bacterial translocation and circulatory disorders (21). Severe sepsis/septic shock patients complicated with neutropenia have a lower risk of receiving mechanical ventilation after PICU admission than those without neutropenia. This partly due to the different risk of ARDS in the two populations. Evidences have shown an increased expression of neutrophil-related genes in the periphery of patients with sepsis and ARDS (22), which suggests that neutrophils play an important role in the early onset of ARDS. The heterogeneity of ARDS pathogenesis is partly determined by circulating neutrophils. Neutropenia may alleviate lung injury and reduce the need of mechanical ventilation. However, in non-neutropenic ARDS, the mechanism of neutrophil-independent lung injury has been discovered (23). The important role of neutrophils in the pathogenesis of ARDS partly explains its lung protection ability.

CRP and PCT levels are related to the severity of infection and disease (24-26), which are of great value to the differential diagnosis and evaluating prognosis of febrile neutropenia after chemotherapy for hematological malignancies (27-29). In our research, the levels of CRP and PCT significantly increased after sepsis onset which paralleled mortality, and further increased when the patients were combined with neutropenia, suggesting that severe neutropenia may worsen the infection. In this study, IL-6 and IL-10 elevated significantly higher in sepsis patients with severe neutropenia, which is consistent with previous discovery $(30,31)$. Notably, the percentage of Tregs also significantly increased in severe neutropenic septic patients, which partly explained the reason for the increase of IL-10. Diepold et al. thought that IL-6 performed well in predicting severity of bacterial infection and sepsis, with high sensitivity and specificity ( $90 \%$ and $85 \%$ ), which is consistent with the research of Smok et al. $(32,33)$. We also observed that the IL-6 and IL-10 levels were significantly different between the survival group and the death group, suggesting that high levels of IL-6 and IL-10 are associated with poor prognosis. Subgroup analysis demonstrated that, regardless of the outcome (survival or dead) of septic patients, IL-6 and IL-10 levels of neutropenic septic patients were always significantly higher than those of nonneutropenic septic patients. It indicates that the levels of pro/anti-inflammatory factors will increase with the onset of neutropenia, and the reason may be that neutropenia could 


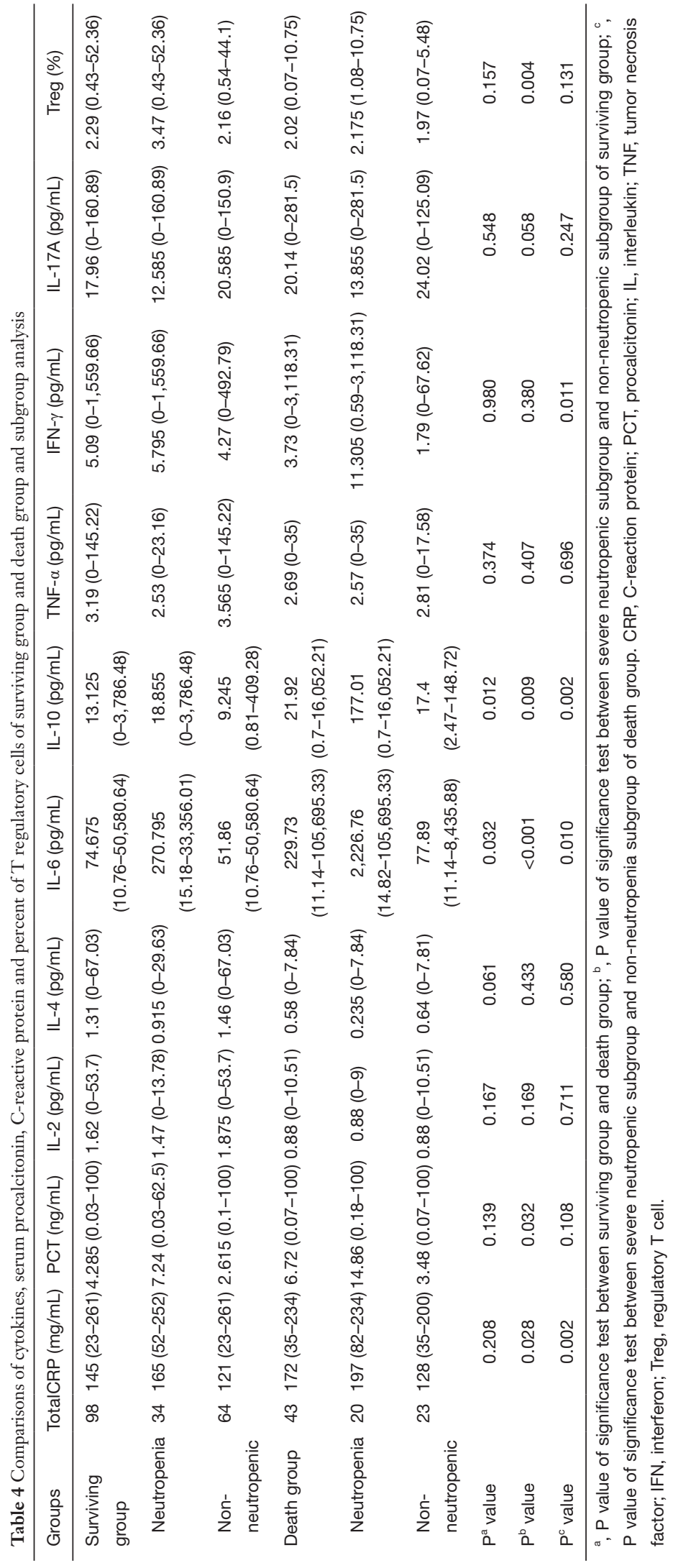


Table 5 IL-6 and IL-10 in evaluating the mortality of sepsis patients and septic neutropenia patients

\begin{tabular}{|c|c|c|c|c|c|}
\hline Groups & AUC & $P$ value & Cut-off value & Sensitivity (\%) & Specificity (\%) \\
\hline IL-6 (pg/mL) & 0.613 & 0.032 & 143.255 & $60.5 \%$ & $62.2 \%$ \\
\hline $\mathrm{IL}-10(\mathrm{pg} / \mathrm{mL})$ & 0.633 & 0.012 & 120.260 & $37.2 \%$ & $90.8 \%$ \\
\hline \multicolumn{6}{|c|}{$\begin{array}{l}\text { Severe neutropenia with severe sepsis/septic } \\
\text { shock }\end{array}$} \\
\hline $\mathrm{IL}-10(\mathrm{pg} / \mathrm{mL})$ & 0.687 & 0.023 & 110.685 & $65.0 \%$ & $79.4 \%$ \\
\hline
\end{tabular}

AUC, area under the curve; IL, interleukin.

weaken the body's response to inflammatory factors (34).

Kitanovski et al. showed that the concentrations of PCT and IL- 6 were the best predictor of the severity of sepsis. In severe sepsis, at a sensitivity level of $100 \%$, the best specificity on day 1 was seen for IL-6 (specificity 57\%) at a cutoff value of $41.8 \mathrm{mg} / \mathrm{L}$ and on day 2 for PCT (specificity $93.2 \%$ ) at a cutoff value of $5.1 \mu \mathrm{g} / \mathrm{L}$ (35). Notably, we found that IL-6 and IL-10 could help identify the risk of death in patients with severe sepsis/septic shock. For patients with severe neutropenia and severe sepsis/septic shock, only IL10 showed a certain predictive value. In our study, apart from IL-6 and IL-10, other cytokines failed to show well diagnostic and predictive value, partly due to the limitation of patient type and small sample size.

\section{Conclusions}

Therefore, severe neutropenia is associated with biomarker features and clinical outcomes of patients with severe sepsis/septic shock. Some cytokines could help predict the prognosis of septic children with neutropenia, and help guide evidence-based integrated treatment. However, underlying diseases may affect cytokine levels. The pathophysiology and immune response of the patients with hematological malignancy included in this study are different from those of patients with non-hematological diseases. Cytokine status of diseases such as tumors is not clear yet, which interferes with clinical judgment. In addition, our research also has many deficiencies, including its retrospective nature, low efficiency, single-center experience, and small sample size. Thus, the results of this retrospective cohort study should be interpreted with caution.

\section{Acknowledgments}

The authors would also like to thank all other physicians in the pediatric intensive care unit division in the Shanghai Children's Medical Center for their support.

Funding: This study was supported by grants from the three-year action plan of promoting clinical skills and innovation ability of municipal hospitals, clinical five new transformation project for special disease of Shanghai Shenkang Hospital Development Center (grant No. 16CR3085B), and the Shanghai Natural Science Foundation of China (grant No. 19ZR1432900).

\section{Footnote}

Reporting Checklist: The authors have completed the STROBE reporting checklist. Available at http://dx.doi. org/10.21037/tp-20-230

Data Sharing Statement: Available at http://dx.doi. org/10.21037/tp-20-230

Conflicts of Interest: All authors have completed the ICMJE uniform disclosure form (available at http://dx.doi. org/10.21037/tp-20-230). The authors have no conflicts of interest to declare.

Ethical Statement: The authors are accountable for all aspects of the work in ensuring that questions related to the accuracy or integrity of any part of the work are appropriately investigated and resolved. The study was performed in accordance with the Declaration of Helsinki (2013 revision) and was approved by the Research 
Ethics Committee of the Shanghai Children's Medical Center of Shanghai Jiao Tong University (Ethical code: SCMCIRBW2020049). Individual consent for this retrospective analysis was waived.

Open Access Statement: This is an Open Access article distributed in accordance with the Creative Commons Attribution-NonCommercial-NoDerivs 4.0 International License (CC BY-NC-ND 4.0), which permits the noncommercial replication and distribution of the article with the strict proviso that no changes or edits are made and the original work is properly cited (including links to both the formal publication through the relevant DOI and the license). See: https://creativecommons.org/licenses/by-nc-nd/4.0/.

\section{References}

1. Singer M, Deutschman CS, Seymour CW, et al. The Third International Consensus Definitions for Sepsis and Septic Shock (Sepsis-3). JAMA 2016;315:801-10.

2. Fleischmann C, Scherag A, Adhikari NK, et al. Assessment of global incidence and mortality of hospital-treated sepsis. current estimates and limitations. Am J Respir Crit Care Med 2016;193:259-72.

3. Urbonas V, Eidukaitė A, Tamulienė I. The predictive value of soluble biomarkers (CD14 subtype, interleukin-2 receptor, human leucocyte antigen-G) and procalcitonin in the detection of bacteremia and sepsis in pediatric oncology patients with chemotherapy-induced febrile neutropenia. Cytokine 2013;62:34-7.

4. Arif T, Phillips RS. Updated systematic review and metaanalysis of the predictive value of serum biomarkers in the assessment and management of fever during neutropenia in children with cancer. Pediatr Blood Cancer 2019;66:e27887.

5. Warnock C. Neutropenic sepsis: prevention, identification and treatment. Nurs Stand 2016;30:51-60.

6. Vincent JL, Rello J, Marshall J, et al. International study of the prevalence and outcomes of infection in intensive care units. JAMA 2009;302:2323-9.

7. Tolsma V, Schwebel C, Azoulay E, et al. Sepsis severe or septic shock: outcome according to immune status and immunodeficiency profile. Chest 2014;146:1205-13.

8. Romagnoli S, Ricci Z, Ronco C. CRRT for sepsis-induced acute kidney injury. Curr Opin Crit Care 2018;24:483-92.

9. Rhodes A, Evans LE, Alhazzani W, et al. Surviving Sepsis Campaign: International Guidelines for Management of Sepsis and Septic Shock: 2016. Intensive Care Med
2017;43:304-77.

10. Colling KP, Banton KL, Beilman GJ. Vasopressors in Sepsis. Surg Infect (Larchmt) 2018;19:202-7.

11. Sharma D, Farahbakhsh N, Shastri S, et al. Biomarkers for diagnosis of neonatal sepsis: a literature review. J Matern Fetal Neonatal Med 2018;31:1646-59.

12. Pierrakos C, Vincent JL. Sepsis biomarkers: a review. Crit Care 2010;14:R15.

13. Holub $M$, Džupová $O$, Růžková $M$, et al. Selected Biomarkers Correlate with the Origin and Severity of Sepsis. Mediators Inflamm 2018;2018:7028267.

14. Song J, Park DW, Moon S, et al. Diagnostic and prognostic value of interleukin-6, pentraxin 3, and procalcitonin levels among sepsis and septic shock patients: a prospective controlled study according to the Sepsis-3 definitions. BMC Infect Dis 2019;19:968.

15. Ríos-Toro JJ, Márquez-Coello M, García-Álvarez JM, et al. Soluble membrane receptors, interleukin 6, procalcitonin and $\mathrm{C}$ reactive protein as prognostic markers in patients with severe sepsis and septic shock. PLoS One 2017;12:e0175254.

16. Jekarl DW, Kim JY, Ha JH, et al. Diagnosis and Prognosis of Sepsis Based on Use of Cytokines, Chemokines, and Growth Factors. Dis Markers 2019;2019:1089107.

17. Kessel A, Bamberger E, Masalha M, et al. The role of $\mathrm{T}$ regulatory cells in human sepsis. J Autoimmun 2009;32:211-5.

18. Cao C, Ma T, Chai YF, et al. The role of regulatory T cells in immune dysfunction during sepsis. World J Emerg Med 2015;6:5-9.

19. Bone RC, Balk RA, Cerra FB, et al. Definitions for sepsis and organ failure and guidelines for the use of innovative therapies in sepsis. The ACCP/SCCM Consensus Conference Committee. American College of Chest Physicians/Society of Critical Care Medicine. Chest 1992;101:1644-55.

20. Hu JD, Yan CH, Feng SZ. Chinese guidelines for the clinical application of antibacterial drugs for agranulocytosis with fever (2016). Zhonghua Xue Ye Xue Za Zhi 2016;37:353-9.

21. Kochanek M, Schalk E, von Bergwelt-Baildon M, et al. Management of sepsis in neutropenic cancer patients: 2018 guidelines from the Infectious Diseases Working Party (AGIHO) and Intensive Care Working Party (iCHOP) of the German Society of Hematology and Medical Oncology (DGHO). Ann Hematol 2019;98:1051-69.

22. Kangelaris KN, Prakash A, Liu KD, et al. Increased expression of neutrophil-related genes in patients with 
early sepsis-induced ARDS. Am J Physiol Lung Cell Mol Physiol 2015;308:L1102-13.

23. Mokart D, Guery BP, Bouabdallah R, et al. Deactivation of alveolar macrophages in septic neutropenic ARDS. Chest 2003;124:644-52.

24. Assicot M, Gendrel D, Carsin H, et al. High serum procalcitonin concentrations in patients with sepsis and infection. Lancet 1993;341:515-8.

25. Yoon SH, Kim EH, Kim HY, et al. Presepsin as a diagnostic marker of sepsis in children and adolescents: a systemic review and meta-analysis. BMC Infect Dis 2019;19:760.

26. Zhou Y, Liu Z, Huang J, et al. Usefulness of the heparinbinding protein level to diagnose sepsis and septic shock according to Sepsis-3 compared with procalcitonin and C reactive protein: a prospective cohort study in China. BMJ Open 2019;9:e026527.

27. García de Guadiana-Romualdo L, Jiménez-Santos E, Cerezuela-Fuentes P, et al. Analyzing the capability of PSP, PCT and sCD25 to support the diagnosis of infection in cancer patients with febrile neutropenia. Clin Chem Lab Med 2019;57:540-8.

28. Luo X, Chen S, Zhang J, et al. Procalcitonin as a marker of Gram-negative bloodstream infections in hematological patients with febrile neutropenia. Leuk Lymphoma 2019;60:2441-8.

29. Phillips RS, Wade R, Lehrnbecher T, et al. Systematic

Cite this article as: Yin F, Xi YL, Wang Y, Li BR, Qian J, Ren H, Zhang J, Tang HZ, Ning BT. The clinical outcomes and biomarker features of severe sepsis/septic shock with severe neutropenia: a retrospective cohort study. Transl Pediatr 2021;10(3):464-473. doi: 10.21037/tp-20-230 review and meta-analysis of the value of initial biomarkers in predicting adverse outcome in febrile neutropenic episodes in children and young people with cancer. BMC Med 2012;10:6.

30. Xia T, Xu X, Zhao N, et al. Comparison of the diagnostic power of cytokine patterns and procalcitonin for predicting infection among paediatric haematology/oncology patients. Clin Microbiol Infect 2016;22:996-1001.

31. Xu XJ, Tang YM, Song H, et al. A multiplex cytokine score for the prediction of disease severity in pediatric hematology/oncology patients with septic shock. Cytokine 2013;64:590-6.

32. Diepold M, Noellke P, Duffner U, et al. Performance of Interleukin-6 and Interleukin-8 serum levels in pediatric oncology patients with neutropenia and fever for the assessment of low-risk. BMC Infect Dis 2008;8:28.

33. Smok B, Domagalski K, Pawłowska M. Diagnostic and Prognostic Value of IL-6 and sTREM-1 in SIRS and Sepsis in Children. Mediators Inflamm 2020;2020:8201585.

34. Dale DC. How I manage children with neutropenia. Br J Haematol 2017;178:351-63.

35. Kitanovski L, Jazbec J, Hojker S, et al. Diagnostic accuracy of lipopolysaccharide-binding protein for predicting bacteremia/clinical sepsis in children with febrile neutropenia: comparison with interleukin-6, procalcitonin, and C-reactive protein. Support Care Cancer 2014;22:269-77. 